\title{
Correction
}

\section{Correction to: Cohomology of the toric arrangement associated with $A_{n}$}

\author{
Olof Bergvall@
}

\section{Correction to: J. Fixed Point Theory Appl. (2019) 21:15 https://doi.org/10.1007/s11784-018-0655-x}

In the version of the article originally published, it was pointed out, in Section 1.1, that Theorem 1.1 could be derived from results in several previous works (namely $[1,2,5,6]$ ). Since then, it has come to our attention that Theorem 1.1 can be more directly derived from either Theorem A(ii) or B(i) of [4]. Both these theorems give descriptions of the cohomology groups of the complement of the toric arrangement associated with the root system $A_{n}$ as representations of the Weyl group $W\left(A_{n}\right)$ in terms of the representation structure of the cohomology groups of the complement of the corresponding hyperplane arrangement (which is known, see, e.g., [3]). Thus, to deduce Theorem 1.1, one simply has to forget the cohomological grading. We would also like to point out that, by remembering the cohomological grading and instead forgetting the representation structure, one can also easily derive Theorem 1.2 from Theorem A(ii) and B(i) of [4].

Open Access. This article is distributed under the terms of the Creative Commons Attribution 4.0 International License (http://creativecommons.org/licenses/by/4. 0/), which permits unrestricted use, distribution, and reproduction in any medium, provided you give appropriate credit to the original author(s) and the source, provide a link to the Creative Commons license, and indicate if changes were made.

Publisher's Note Springer Nature remains neutral with regard to jurisdictional claims in published maps and institutional affiliations.

The original article can be found online at https://doi.org/10.1007/s11784-018-0655-x. 


\section{References}

[1] Gaiffi, G.: The actions of $S_{n+1}$ and $S_{n}$ on the cohomology ring of a Coxeter arrangement of type $A_{n-1}$. Manuscr. Math. 91(1), 83-94 (1996)

[2] Getzler, E.: Operads and moduli spaces of genus 0 Riemann surfaces. In: Dijkgraaf, R.H., Faber, C.F., van der Geer, G.B.M. (eds.) The Moduli Space of Curves. Progress in Mathematics, vol. 129, 199-230. Birkhäuser, Boston (1995)

[3] Lehrer, G.: On the Poincare series associated with Coxeter group actions on complements of hyperplanes. J. Lond. Math. Soc. 36(2), 275-294 (1987)

[4] Lehrer, G.: A toral configuration space and regular semisimple conjugacy classes. Math. Proc. Camb. Philos. Soc. 118(1), 105-113 (1995)

[5] Mathieu, O.: Hidden $\Sigma_{n+1}$-Actions. Commun. Math. Phys. 176(2), 467-474 (1996)

[6] Robinson, A., Whitehouse, S.: The tree representation of $\Sigma_{n+1}$. J. Pure Appl. Algebra 111(1-3), 245-253 (1996)

Olof Bergvall

Matematiska Institutionen

Uppsala Universitet

Box 48075106

Uppsala

Sweden

e-mail: olof . bergvall@math.uu.se 